zu Themen rund um das Thema Sexualität bei verschiedenen Quellen.

Beide Geschlechter bevorzugen nach wie vor Gespräche mit erwachsenen Vertrauenspersonen, ebenso den Austausch mit Gleichaltrigen. Stehen diese als Ansprechpartner zu wenig zur Verfügung, dienen Medien zur Kompensation von Wissenslücken. Dazu nutzen Jugendliche Aufklärungsmedien sowie soziale Netzwerke oder pornografische Darstellungen. Auch die schulische Aufklärung, die in Deutschland flächendeckend stattfindet, nimmt einen großen Stellenwert ein.

Jugendliche handeln meist verantwortungsbewusst, partnerschaftlich und verhüten heutzutage so sicher wie keine Generation zuvor. Ihr erstes Mal erleben sie meist zwischen dem siebzehnten und zwanzigsten Lebensjahr [4]. Für die meisten von ihnen wäre eine Schwangerschaft eine „Katastrophe“, deshalb verhüten sie sicherer als die Gruppe der jungen Erwachsenen zwischen 25 und 35 Jahren. Die Anzahl der minderjährigen Schwangeren ist in Deutschland sehr gering. Partnerschaftliches Handeln, gegenseitige Verantwortung und Treue sind für die Mehrheit der Jugendlichen ein Muss, wenn sie sexuell aktiv sind. Die Geschlechter haben sich weitestgehend aneinander angenähert, und Mädchen fühlen sich emanzipiert. Die meisten Eltern unterstützen es, wenn Jugendliche partnerschaftliche Sexualität ausleben, zum Beispiel gemeinsam übernachten.

\title{
KURZ GEFASST
}

\author{
1 Jugendliche verhüten beim Sex sicherer als die Gruppe der jungen \\ Erwachsenen zwischen 25 und 35 Jahren. \\ 2 Der Konsum von Pornografie lässt meist nach, wenn sich \\ Jugendliche in einer festen Partnerschaft befinden. \\ 3 Sexualitätsbegleitung hilft unter anderem, Mythen wie \\ „Je größer der Penis, desto besser der Sex“ zu korrigieren.
}

Pornografie in Berührung, aber in der Regel ein Jahr später als die Jungen. Das Durchschnittsalter der ersten Berührung mit Pornografie liegt bei 14,2 Jahren.

Mädchen bewerten Pornografie eher als uninteressant, lehnen sie ab oder finden sie abstoßend. Jungen tauschen sich unter Gleichaltrigen gerne über „öde“, „krasse“, „perverse“, „,eklige“ Pornografie aus. Sie begreifen diese dann weniger als sexuelle Stimuli, sondern nutzen sie im Sinne von Kommunikation, Provokation, Mutproben und Rückversicherung der eigenen Normalität. Manchmal schauen sich Jugendliche Pornos an, um sich über Details zu sexuellen Praktiken zu informieren [1].

Kontakt mit Pornografie findet bei Jugendlichen trotz strafrechtlicher Schutzbestimmungen statt. Via Internet ist heutzutage vieles möglich, sodass eine Medienregulierung seitens der Erwachsenen nur sehr begrenzt funktioniert. Der erste Kontakt findet bei knapp 50\% der Jugendlichen ungewollt statt, meistens als Zufallsfund, wenn sie im Internet unterwegs sind. Laut Döring trägt Medi-

\section{Partnerschaftliches Handeln, gegenseitige Verantwor- tung und Treue sind für die Mehrheit der Jugendlichen ein Muss, wenn sie sexuell aktiv sind.}

\section{Umgang mit Pornografie}

Jugendliche bewegen sich im Internet und stoßen dabei auf pornografische Darstellungen. Das kann zufällig und ungeplant passieren, aber es werden auch Geschichten, Zeichnungen, Fotos und Videos mit sexuellen Inhalten gezielt ausgesucht. Dabei sind die Jungen aktiver als die Mädchen. Mädchen kommen zwar auch mit enbildung entscheidend dazu bei, die Risiken gegen die Pornografisierung von Jugendlichen aller Geschlechter zu minimieren [2].

\section{Reaktion auf Pornos: von Erregung bis Belustigung}

Laut einer Studie der Universität Münster und Hohenheim (2017) konsumie- ren Jugendliche Pornos mehrheitlich zu Hause oder bei einem Freund. Schauen sie sich Pornos bewusst an, etwa um sich sexuell zu erregen, sind sie lieber alleine. Die Erlebnisse mit pornografischen Inhalten sind unterschiedlich. Sie reichen von Gleichgültigkeit (23\%), Unwichtigsein (22\%), Erregung (18\%) hin bis zur Belustigung (14\%). Wenn Jugendliche alleine sind und sich bewusst Pornos anschauen, überwiegt die Erregung. Sind hingegen andere Personen im Raum äußern sie sich vornehmlich belustigt. $60 \%$ der Jugendlichen, die sich beim Erstkonsum unwohl gefühlt haben, sprechen mit niemandem darüber.

Der Konsum lässt meistens nach, wenn sich Jugendliche in einer festen Partnerschaft befinden. Sexuelle Erfahrungen im realen Leben scheinen dann interessanter zu sein als digitale Erlebnisse.

In der Regel sind Jugendliche in der Lage, zwischen ihren eigenen sexuellen Lebenswelten und fiktionalen Pornosettings zu unterscheiden. Dennoch ist eine gute Medien- und Sexualerziehung, die auch die Nutzung von Pornografie mit einbezieht, bedeutsam. Das Gleiche gilt für die Thematisierung von Cypermobbing und Sexting. Sexting bedeutet, mit ausgewählten Personen bewusst Bilder und persönliche Daten mit sexuellen Bezügen digital (zum Beispiel via Internet oder WhatsApp) auszutauschen.

\section{Umgang mit Medien und Smartphones}

Das Smartphone hat die Nutzung des Internets per Computer, Laptop oder Tablets weitestgehend abgelöst. Jugendliche benutzen das Smartphone als Alltagsgegenstand, um sich zu informieren, andere zu kontaktieren und sich in sozialen 
Netzwerken zu inszenieren. Differenzierte Studien zu dem Thema stehen noch aus. Es ist aber davon auszugehen, dass diese Art der veränderten Kommunikation Auswirkungen auf die Lebenswelt der Jugendlichen und letztendlich auf die Beziehungsgestaltung und Sexualität hat.

Das Schreiben per WhatsApp und die Veröffentlichung persönlicher Befindlichkeiten ersetzt einen Großteil von persönlichen Kontakten in Gesprächen von Angesicht zu Angesicht oder per Telefon. Mimik und Tonfall entfallen bei dieser Art der Kommunikation. Die Distanz zum „Ich“ und dem Gegenüber vergrößert sich, wird aber nicht bewusst als solches wahrgenommen. Jugendliche vermissen andere Wege der Kommunikation nicht, weil sie anders als früher mit alltäglichem Medienkonsum aufwachsen.

In der Jugendarbeit bemerken wir aber auch, dass die Kommunikation per WhatsApp und in sozialen Netzwerken viele Vorteile hat. Es kann zur schnellen Kontaktaufnahme und zu spontanen Mitteilungen über Gefühle kommen. Das kann Einzelnen, gerade in der pubertären Phase, Sicherheit und Ich-Stärke vermitteln. Sie fühlen sich verstanden und nicht alleine mit ihren Fragen und Problemen. Aber es gibt auch größere Interpretationsspielräume. Wenn Jugendliche anderen ein Foto von sich schicken, in dem sie sich sexy inszenieren, kann dieses von den einen als cooles Bild, von anderen aber als Einladung zum Sex verstanden werden. Dazu ein Beispiel aus meiner Beratungspraxis: Maria, ein Mädchen mit Förderbedarf, schickte einem befreundeten Jungen aus der Klasse ein Foto von sich, bei dem sie nur ein Top und einen Slip trug. Sie hatte das Smartphone neu und fotografierte alles, was sie schön und spannend fand. Dann versandte sie die Fotos an Personen, die sie nett fand. Der Junge, der das Foto erhalten hatte, fühlte sich aber sexuell belästigt und war zutiefst verunsichert. Die Freundschaft kam ins Wanken, weil er sich beschämt fühlte. Maria verstand die Reaktion des Jungen nicht, denn sie fand es normal, ein „nettes Foto“ von sich zu verschicken. Sie sah keinen Unterschied im Tragen von Unterwäsche oder Badesachen.

\section{Soziale Kontrolle durch soziale Medien}

In unseren Veranstaltungen beobachten wir, dass soziale Medien auch soziale Kontrollen begünstigen. Jugendliche setzten sich dem freiwillig und meist unbewusst aus. Das schützt sie dennoch nicht vor inneren Konflikten. Beispiel: Luis schreibt seinem Freund aus der Disco eine WhatsApp-Nachricht, in der er mitteilt, dass er gerade ein süßes Mädchen kennengelernt hat. Durch die Veröffentlichung kann sich der innere Druck erhöhen, dass an dem Abend nun auch „sexuell etwas passieren muss“, um nicht das Gesicht zu verlieren.

Je mehr Privates unbedacht veröffentlicht wird, desto größer kann der Leistungsdruck werden. Das Zarte der Annäherung entfällt dann, ebenso die Zeit und der Raum, um erst mal zu schauen, was man eigentlich will. Somit fehlt die Chance zur Selbstvergewisserung und größtmöglichen Selbstbestimmung ohne sozialen Druck und unabhängig von den Reaktionen und Erwartungen anderer. Das kann dann dazu führen, dass Jugendliche weniger Selbstbewusstsein entwickeln, unsicher im sozialen Agieren sind und weniger Zutrauen in sich selbst und die eigenen Handlungen haben.

\section{Körperbilder in Medien}

Medien haben Einfluss auf das eigene Körperbild, zum Beispiel in Form von Werbeplakaten, Sportübertragungen, Musikvideos etc. Für Jungen steht ein sportlicher, muskulöser Körper an erster Stelle. Dafür nehmen sie sich Zeit und gehen zum Beispiel ins Fitness-Studio und treiben viel Sport. Die meisten fühlen sich in ihrem Körper wohl (72\%). Mädchen fällt es schwerer, den eigenen Körper so anzunehmen, wie er ist. Nur knapp die Hälfte der Mädchen stimmt der Aussage „Ich fühle mich wohl in meinem Körper“ zu. Jede Dritte ist mit ihrem Körpergewicht unzufrieden. Hingegen hat das Stylen einen gewichtigen Anteil für ein positives Körpergefühl (71\%) [4].

\section{Hilfreiche Sexualitätsbegleitung}

Die mannigfaltigen Kommunikationsund Informationsmöglichkeiten fördern gesellschaftlich und medial verbreitete Mythen wie „Zum guten Sex gehört ein gleichzeitiger Orgasmus“ oder „Je größer der Penis, desto besser der Sex“. Mythen halten sich hartnäckig und beeinflussen unsere Haltung und auch die von Heranwachsenden. In einer Zeit, in der Kinder und Jugendliche vielfältig mit dem Thema Sexualität konfrontiert sind, kommen Erwachsene nicht umhin, sich aus pädagogischer, beraterischer und therapeutischer Sicht mit Kinder- und Jugendsexualität zu beschäftigen.

\section{Aufklärung in Zeiten hartnäckiger Mythen}

Leon, 16 Jahre, wendet sich an mich in der Onlineberatung, weil sein Penis für sein Alter zu klein sei. Er berichtet, dass er seit einem halben Jahr eine feste Freundin habe und dass ihr gemeinsames erstes Mal ansteht. Er hat Angst, dass der Geschlechtsverkehr nicht funktionieren wird.

Nina, 18 Jahre, schläft drei- bis viermal in der Woche mit ihrem Freund. Sie berichtet, dass sie auf den Geschlechtsverkehr mit ihm nicht verzichten möchte, weil es für sie ein schönes Gefühl sei, ihn so nahe bei sich zu spüren. Aber körperlich empfindet sie darüber hinaus nichts. Beim Vorspiel wird Ihre Vulva feucht, aber ein lustvolles Körpergefühl will sich dabei nicht so recht einstellen. Beide leiden unter dieser für sie unbefriedigend erlebten Situation. Ninas Freund hat inzwischen immer weniger Lust und vermeidet sexuelle Situationen. Das verletzt Nina, und sie gibt sich die Schuld dafür.

Die hier exemplarisch beschriebenen Fälle sind keine Seltenheit. Suchen Klienten eine Beratungsstelle rechtzeitig auf, helfen meist wenige klärende Gespräche. Dazu gehört es, Mythen aufzulösen und Körper- sowie Sexualinformationen zu vermitteln. Leon würde die Information helfen, dass es beim Sex nicht auf die Größe des Penis ankommt. Dass sich ein kleiner Penis mit einer Erektion stark vergrößert und dass die Vagina so beschaffen ist, dass sie sich beim Geschlechtsverkehr an die Größe des Penis anpasst. Für Nina und ihren Freund kann 
man die Situation entkrampfen, indem man ihnen Sexualinformationen zu den unterschiedlichen männlichen und weiblichen Reaktionen gibt. Auch Anregungen, wie sie ihren gemeinschaftlichen Sex verbessern können, wären hilfreich.

\section{Weshalb ist Sexualitätsbegleitung wichtig?}

Sexualität kann eine Ressource sein und Menschen Wohlbefinden, Kraft und Freude schenken. Sie ist aber auch störanfällig und hat dann nicht nur Auswirkungen auf die Sexualität, sondern auch auf die Beziehung, die partnerschaftliche und individuelle Bedürfnisgeschichte sowie auf das eigene Selbstwertgefühl. Gefühle wie Liebe und sexuelles Begehren hängen zusammen und von vielerlei Faktoren ab. Ein dauerhaft erlebter Mangel an liebevoller Zuwendung und verbindlichen Beziehungen kann das Liebesfühlen stark beeinträchtigen.

Im Alltag und in vielen Behandlungen sowie begleitenden Settings wird jedoch nicht über Sexualität gesprochen. Sie wird meist auch in den gängigen Ausbildungen ausgespart. Einen Umdenkprozess in Behandlungen und Beratungen einzuleiten, erscheint lohnenswert.

Voraussetzung dafür ist es, nicht immer nur die problemorientierte Seite des

- INFORMATION

\section{Sexualität: auch bei Erwachsenen ein Thema}

Lange Zeit wurde übersehen, dass es Rund um das Thema der Sexualität einen Bedarf nach Wissen, Rat und Beratung jenseits von klassifizierten Störungen gibt. Im alltäglichen sexuellen Erleben kommt es immer wieder zu konflikthaften, belastenden Situationen, woraus der Wunsch nach einer Beratung oder einem Gespräch erwächst. Nicht nur den Kindern und Jugendlichen mangelt es hier an fachlich qualifizierten Ansprechpartnern. Der größte Teil der Erwachsenen sucht (erst) dann eine Sexualberatungsstelle auf, wenn sich Störungen bereits manifestiert haben. Viele haben sich zuvor mit sexuellen Problemen und Fragestellungen an Behandelnde gewandt, die ihnen nur unzureichend weitergeholfen haben.

Sexualstörungen, zum Beispiel Orgasmusschwierigkeiten oder Erektionsstörungen, entstehen mehrheitlich durch psychische und nicht durch physische Ursachen. Medizinische Hilfen tragen nur selten dazu bei, das sexuelle Problem zu lösen. Ähnlich wie jugendlichen Patienten können Sie auch Erwachsenen helfen, Sexuelles zu thematisieren, indem Sie ihnen - unter Berücksichtigung des Datenschutzes und der Schweigepflicht - von anderen Fällen berichten.

Im Rahmen einer Diagnostik und eines Behandlungskonzepts sollten auch körperliche Ursachen und ihre Auswirkung auf die Sexualität besprochen werden. So ist es zum Beispiel bekannt, dass Diabetes auch zu sexueller Lustlosigkeit führen kann.

Tipp: Sowohl Jugendliche als auch Erwachsene können sich bundesweit bei pro familia Beratungsstellen zu allen sexualitätsund partnerschaftsbezogenen Themen beraten lassen. Es gibt dort auch kostenloses Informationsmaterial. Weitere Informationen unter: www.profamilia und www. sextra.de
Sexuellen in den Fokus zu nehmen und das Thema (mehr) mit entsprechender Reflexions- und Handlungskompetenz zu versehen. Menschen, die sich in eine Behandlung begeben, tun es dann, wenn Störungen bereits aufgetreten sind, sich ein Problem bereits manifestiert hat. Besser wäre es, wie bei Nina oder Leon, wenn sie im Vorfeld eine Ansprechperson gehabt hätten, die ihnen ein $\mathrm{Ge}$ sprächsangebot gemacht und über Sexualität so selbstverständlich gesprochen hätte wie über andere Dinge auch.

Sexualität wird auch durch Gelerntes beeinflusst. Sie ist in Teilbereichen veränderbar und gestaltbar. Sexualaufklä- 
rung kann dazu beitragen, dass Jugendliche weniger schlechte sexuelle Erfahrungen machen, zum Beispiel weil sie sich dann sicher verhütet fühlen und sich dann entspannter auf das sexuelle Erleben einlassen können. Frühzeitig aufzuklären und zu beraten, hilft dem Jugendlichen, kompetenter zu handeln und zu reflektieren. Störungen können frühzeitig erkannt und mithilfe entsprechender Beratungen und Behandlungen vermieden werden.

\section{Was können Sie tun?}

Sprechen Sie in Ihren Behandlungen auch über das Thema körperliche Entwicklung und Sexualität. Beziehen Sie Themen wie Körper, Zufriedenheit mit dem Aussehen und Sexualität in die Anamnese ein. Sprechen hilft - und zwar auf zweierlei Art: Einerseits ist es einfacher, sich selbst ein Problem einzugestehen und dies zu bearbeiten. Erkenntnis ist auch im Therapieverlauf der erste wichtige Schritt zur Veränderung. Andererseits wird Kommunikation ermöglicht und das Sprechen über Sexualität gefördert.

Versuchen Sie Vertrauen zum Jugendlichen aufzubauen und diese Themen als natürlich und normal anzusehen. Versichern Sie ihnen, dass diese Gespräche vertraulich sind. Bedrängen Sie die Jugendlichen nicht, sondern geben Sie ihnen die Möglichkeit, eigene Themen zu

INFORMATION

\section{Sexuelle Selbstbestimmung} fördern, sexuelle Bildungsprozesse begleiten

Das ISP (institut für Sexualpädagogik) ist das führende Fachinstitut im Bereich sexuelle Bildung und Sexualpädagogik. Der Zusammenschluss sexualpädagogisch tätiger Personen hat die gemeinsame Idee entwickelt, sexuelle Bildung zu fördern und zu professionalisieren. Seit 1988 bildet das ISP Fachkräfte in Deutschland, Österreich, Südtirol und in der Schweiz aus. Es können fortlaufende Weiterbildungen, einzelne Seminare, Vorträge oder Workshops besucht oder gebucht werden. Das ISP unterstützt auch bei der Konzeptentwicklung und bietet Praxisberatung an.

Informationen finden Sie unter: https://www.isp-sexualpaedagogik.org formulieren. Um diese zur Sprache zu bringen, helfen beispielhafte Geschichten von anderen Jugendlichen.

Kurbeln Sie den Prozess des Bewusstwerdens an. Bringen Sie Sexuelles zur Sprache, dort wo Worte fehlen. Das kann durchaus eine knifflige Angelegenheit sein. Daher ist es sinnvoll, sich zuvor selber in diesen Bereichen fortzubilden. In schwierigen Situationen kann die Einbeziehung einer Beratungsstelle nützlich sein. Auch Heilpraktiker können sich dort unterstützen und beraten lassen, um die Impulse dann ins Gespräch mit den Jugendlichen einzubringen. Einschlägige Institutionen und Ausbildungszentren bieten zu allen sexualitätsbezogenen Themen Fort- und Weiterbildungen an.

Eine hilfreiche Sexualitätsbegleitung bezieht den Körper als wesentlichen Bestandteil von sexuellem Erleben mit ein. Sexualität ist im Körper immer spürbar und wird vor allem körperlich ausgedrückt. Durch langes Sitzen im Schulunterricht und Medienkonsum schenken Jugendliche ihrem Körper weniger Aufmerksamkeit. Das führt nicht nur zu körperlichen Beeinträchtigungen, sondern auch zu einem Mangel an psychischem Wohlbefinden sowie Einschränkungen in der motorischen und kognitiven Entwicklung. Behutsame Angebote zur Körperwahrnehmung und/oder zur Entspannung verbessern das körperliche Wohlbefinden und tragen dazu bei, dass sich Körper, Geist und Emotionen mehr im Einklang miteinander befinden.

\section{Zutrauen und Kompetenzen fördern}

Vertrauen, Zutrauen, Sprach-, Kommunikations- und Medienkompetenz stärken das junge Individuum und seine Sexualität. Medien liefern Jugendlichen bereits vorpubertär „Drehbücher“ über sexuelles Verhalten im Erwachsenenalter. Eigene Träume, Unsicherheit und Angst prallen auf öffentliche Inszenierungen von sexuellen Handlungen. Jugendlichen ist die Benutzung von Medien vertraut, und sie nutzen sie sinnvoll. Dennoch benötigen sie bisweilen Unterstützung darin zwischen digitalen und realen sowie zwischen jugendlichen und erwachsenen
Lebenswelten zu unterscheiden. Mediale Einflussfaktoren sind nicht von der Hand zu weisen, doch die Annahme monokausaler Wirkungsmechanismen konnte in wissenschaftlichen Untersuchungen nicht bestätigt werden.

Persönliche Kommunikation auch über Sexuelles erübrigt sich nicht durch Mediennutzung. Konkrete Menschen, Erlebnisse, Lern- und Erfahrungsgeschichten haben in der Wirkung einen deutlich höheren Stellenwert. Jugendliche tauschen sich in ihrer Peergruppe (zum Beispiel in der Clique) über sexuelle Fragen aus. Sind erwachsene Vertrauens-und Ansprechpersonen vorhanden, gibt das Jugendlichen Orientierung und Sicherheit im Entwicklungsprozess. Massive Störungen im Sozialisationsprozess, zum Beispiel Gewalterfahrungen), benötigen andere Wege der Begleitung und Behandlung.

Dieser Artikel ist online zu finden: http://dx.doi.org/10.1055/a-0626-5181

\section{Verwendete Literatur}

[1] Bauer Media Group (Hrsg.). Bravo Dr. Sommer Studie 2016. Hamburg; 2016

[2] Döring N. Alle Jugendlichen schauen Pornografie. In: 11 Gründe, mit Jugendlichen über Sex zu sprechen. Hannover: LJS Niedersachsen; 2017. S. 20-21

[3] Döring N. Pornografische Kompetenz. Definition und Förderung. In: Deutsche Gesellschaft für Sexualforschung (Hrsg.). Zeitschrift Sexualforschung $1 / 2011$

[4] Heßling A, Bode H. Jugendsexualität 2015. Die Perspektive der 14- bis 25-jährigen. Ergebnisse einer repräsentativen Wiederholungsbefragung.

\section{HP Beate Martin}

E-Mail: b.martin@isp-sexualpaedagogik.org

Beate Martin ist Heilpraktikerin für Psychotherapie und Dozentin des Instituts für Sexualpädagogik, Projektleiterin der Weiterbildung „Sexualität und Behinderung“, sexualpädagogische Fachkraft bei pro familia Münster, Autorin vielfältiger sexualpädagogischer Bücher und Fachartikel. Sie ist Gesprächpsychotherapeutin (Gesellschaft für wissenschaftliche Gesprächspsychotherapie, GWG Köln), Sexualtherapeutin (Deutsche Gesellschaft für Sexualwissenschaft, DGfS), NLP Practitioner (DGNLPT) und zertifizierte Onlineberaterin. 